\title{
Prevalence and associated knowledge of hepatitis B infection among healthcare workers in Freetown, Sierra Leone
}

\author{
Yu-Ling Qin ${ }^{1 \dagger}$, Bo Li ${ }^{1 \dagger}$, Yue-Su Zhou ${ }^{1 \dagger}$, Xin Zhang ${ }^{1}$, Lei Li ${ }^{1}$, Bing Song ${ }^{1}$, Peng Liu', Yue Yuan ${ }^{1}$, Zhong-Peng Zhao²,

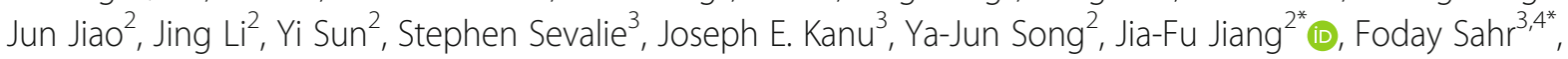 \\ Tian-Jun Jiang ${ }^{1 *}$ and Chinese Military Medical Experts Group in Sierra Leone
}

\begin{abstract}
Background: Hepatitis B virus (HBV) is considered highly prevalent in West Africa. However, major gaps in surveillance exist in Sierra Leone. Although healthcare workers (HCWs) are at high risk for HBV infection, little is known about the prevalence and knowledge of hepatitis B among HCWs in Sierra Leone.

Methods: A cross-sectional study of all HCWs at the No. 34 Military Hospital located in Freetown, Sierra Leone, was conducted from March 20 to April 10, 2017. Whole blood was collected and screened for HBV markers using a onestep rapid immunochromatographic test with positive samples tested for HBV DNA. Additionally, questionnaires assessing self-reported knowledge of HBV infections were administered to all participants. Data were processed and analyzed using SPSS (version 17.0) software.

Results: A total of 211 HCWs were included in this study with a median age of 39.0 years (range: 18-59). Of the participating HCWs, 172 (81.5\%) participants were susceptible (all markers negative), 21(10.0\%) were current HBV ( $\mathrm{HBs} A g$ positive) and nine (4.3\%) were considered immune because of past infection (HBsAg negative and anti-HBC positive; anti-HBs positive). Additionally, nine (4.3\%) participants displayed immunity to the virus as a result of prior hepatitis B vaccination (only anti-HBs positive). Of the $21 \mathrm{HCWs}$ with positive HBsAg, 13 (61.9\%) had detectable HBV DNA. There was a significantly lower risk for current HBV infection among HCWs older than 39 years (OR 0.337, $p=$ 0.046). In addition, only 14 (6.6\%), 73 (34.6\%) and 82 (38.9\%) participants in this survey had adequate knowledge about the clinical outcome, routes of transmission, and correct preventive measures of HBV infection, respectively.

Conclusions: HCWs in Sierra Leone lacked adequate knowledge of the hepatitis B virus. Additionally, the low coverage rate of hepatitis $B$ vaccination among HCWs fails to meet WHO recommendations, leaving many of the sampled HCWs susceptible to infection. This study reaffirms the need for more intensive training for HCWs in addition to strengthening vaccination programmes to protect HCWs against HBV in Sierra Leone.
\end{abstract}

Keywords: Hepatitis B virus, Healthcare workers, Prevalence, Sierra Leone

\footnotetext{
*Correspondence: jiangjf2008@gmail.com; fodaysahr1@gmail.com; aba302@163.com

†Yu-Ling Qin, Bo Li and Yue-Su Zhou contributed equally to this work.

${ }^{2}$ Beijing Institute of Microbiology and Epidemiology, Beijing, China

${ }^{3}$ No. 34 Military Hospital, Wilberforce, Freetown, Sierra Leone

'Beijing 302 Hospital, Beijing, China

Full list of author information is available at the end of the article
}

(c) The Author(s). 2018 Open Access This article is distributed under the terms of the Creative Commons Attribution 4.0 International License (http://creativecommons.org/licenses/by/4.0/), which permits unrestricted use, distribution, and reproduction in any medium, provided you give appropriate credit to the original author(s) and the source, provide a link to the Creative Commons license, and indicate if changes were made. The Creative Commons Public Domain Dedication waiver (http://creativecommons.org/publicdomain/zero/1.0/) applies to the data made available in this article, unless otherwise stated. 


\section{Background}

Hepatitis B virus (HBV) infection is a major threat to public health globally. According to the World Health Organization (WHO), approximately 257 million people are infected with chronic HBV [1]. Infection with HBV can increase the risk of death from liver cirrhosis and hepatocellular carcinoma, which is the sixth most common cancer and the third cause of cancer death worldwide [2]. The WHO reported that hepatitis $B$ prevalence is the highest in the western Pacific region and Africa, where 6.2 and $6.1 \%$ of the adult population are infected, respectively [1]. Sierra Leone, located in West Africa, is considered a high-endemic area. A study conducted among primary school children, in 1998, detected HBsAg in $18 \%$ of the children tested [3]. In 2005, a study showed a $6.2 \%$ seroprevalence of hepatitis B among pregnant women of middle and high socio-economic class in Sierra Leone [4]. Recently, a study screening blood donor candidates for blood-borne pathogens found a HBsAg prevalence of $15 \%$ in men and $13 \%$ in women from a single hospital in Tonkolili Province, Sierra Leone [5]. These results illustrate the serious public health risks that hepatitis B poses in Sierra Leone. In addition, HBV continues to be an understudied topic in Sierra Leone. Due to limited resources, little has been done to combat hepatitis B in Sierra Leone despite the suspected high burden of disease. Although the hepatitis $B$ vaccine was introduced for 6 to 14 week-old children, there are currently no active programs administering the vaccine at birth. Furthermore no coordinated $\mathrm{HBV}$ vaccination program been put in place to prevent infection among the adult population [6].

Healthcare workers (HCWs) are considered a high-risk group for HBV infection due to occupational exposure to blood-borne pathogens. Previous studies in Africa found high HBV infection and exposure rates (roughly 10\%) in HCWs in South Africa and Nigeria. Worldwide, approximately 2 million HCWs are infected with HBV through sharp injury [7-9]. This study was designed to evaluate the prevalence of HBV markers in HCWs as well as to assess their knowledge of HBV infection and prevention in Sierra Leone.

\section{Methods}

\section{Study area and sample population}

A cross-sectional study was conducted at the No. 34 Military Hospital in Freetown, Sierra Leone from March 20 to April 10, 2017. This hospital serves as a medical center for the Republic of Sierra Leone Armed Forces in addition to also being a teaching and general hospital. A total of HCWs, including medical doctors, nurses and other non-clinical health workers, were studied and written, informed consent was obtained from each subject.

\section{Laboratory detection of hepatitis B virus}

Blood samples were drawn from the antecubital vein of the 211 participants by phlebotomists of the No. 34 Military Hospital clinical laboratory then centrifuged for $5 \mathrm{~min}$ at $12,000 \mathrm{~g}$ at room temperature. Serological tests were performed to detect five markers including $\mathrm{HBsAg}$, anti-HBs, $\mathrm{HBeAg}$, anti-HBe and anti-HBc, by using a one-step rapid immunochromatographic test (Shanghai Kehua Bio-engineering Co., Ltd., Shanghai, China). Test results were interpreted and reported as positive or negative based on the manufacturer's instructions.

Viral deoxyribonucleic acid (DNA) was extracted using the QIAamp DNA Blood Mini Kit (QIAGEN, Germantown, MD, USA) according to the manufacturer's instructions. HBV DNA was amplified using real-time PCR (qPCR) (Light Cycler Software Version 4.1, Roche Diagnostics, Penzburg, Germany) HBV DNA assay kits (Sansure Biotech, Changsha, China) in all HBsAg positive samples.

\section{Design and administration of the questionnaire}

Data were collected using a self-administered questionnaire, which was developed after reviewing relevant research [10-12]. The survey had questions on socio-demographic characteristics, knowledge of HBV clinical outcome, route of transmission and preventive measures against hepatitis B infection, previous infection, and previous vaccination history. No vaccinations were offered to HCWs as part of this study. Seventeen questions had binary (yes or no) responses, and three multi-items questions focusing on the clinical outcome of HBV infection, route of transmission and proper preventive measures had only one correct answer. Each study participant was expected to complete the questionnaire.

\section{Statistical analysis}

The data obtained from the questionnaire and the results of the laboratory test were analyzed using SPSS (version 17.0, SPSS Inc. Chicago, IL). The response for each question was given a score of one and zero indicating a right or wrong answer was provided, respectively. Then we summed and graded the total scores as 'poor', 'intermediate' or 'adequate' level for each study participant based on the distribution located in the tri-sectional quantiles of the grouped data array. The Pearson Chi-square test was used to determine the relationships between participant characteristics and HBV marker detection rates. Multivariable factor analysis for current HBV infection was 
Table 1 Prevalence of five hepatitis B virus serological markers by socio-demographic characteristics of the study participants

\begin{tabular}{|c|c|c|c|c|c|c|c|c|c|c|c|}
\hline Characteristic & $\begin{array}{l}\text { Tested } \\
\text { no. (\%) }\end{array}$ & $\begin{array}{l}\text { HBsAg (+) } \\
\text { no. }(\%)\end{array}$ & $P$ value & $\begin{array}{l}\text { HBsAb (+) } \\
\text { no. }(\%)\end{array}$ & $P$ value & $\begin{array}{l}\text { HBeAg }(+) \\
\text { no. }(\%)\end{array}$ & $P$ value & $\begin{array}{l}\text { HBeAb (+) } \\
\text { no. (\%) }\end{array}$ & $P$ value & $\begin{array}{l}\text { HBcAb (+) } \\
\text { no. }(\%)\end{array}$ & $P$ value \\
\hline \multicolumn{12}{|l|}{ Age (y) } \\
\hline$\geq 39 y$ & $112(53.1)$ & $6(5.4)$ & $0.021^{*}$ & $7(6.3)$ & 0.342 & $0(0.0)$ & 0.469 & $7(6.3)$ & $0.027^{*}$ & $10(8.9)$ & 0.066 \\
\hline$<39 y$ & 99 (46.9) & $15(15.2)$ & & $3(3.0)$ & & $1(1.0)$ & & $16(16.2)$ & & $18(18.2)$ & \\
\hline \multicolumn{12}{|l|}{ Gender } \\
\hline Male & $108(51.2)$ & $10(9.3)$ & 0.820 & $7(6.5)$ & 0.333 & $0(0.0)$ & 0.488 & $11(10.2)$ & 0.826 & $13(12.0)$ & 0.686 \\
\hline Female & $103(48.8)$ & $11(10.7)$ & & $3(2.9)$ & & $1(1.0)$ & & $12(11.7)$ & & $15(14.6)$ & \\
\hline \multicolumn{12}{|l|}{ Working experience } \\
\hline$\geq 9 y$ & $113(53.6)$ & $8(7.1)$ & 0.168 & $7(6.2)$ & 0.345 & $0(0.0)$ & 0.464 & $8(7.1)$ & 0.076 & $10(8.8)$ & 0.066 \\
\hline$<9 y$ & $98(46.4)$ & $13(13.3)$ & & $3(3.1)$ & & $1(1.0)$ & & $15(15.3)$ & & $18(18.4)$ & \\
\hline \multicolumn{12}{|l|}{ Education level } \\
\hline High school & 39 (18.5) & $6(15.4)$ & 0.365 & $1(2.6)$ & 0.645 & $0(0.0)$ & 0.873 & $5(12.8)$ & 0.807 & $7(17.9)$ & 0.601 \\
\hline Diploma certificate & $166(78.7)$ & $14(8.4)$ & & $9(5.4)$ & & $1(0.6)$ & & $17(10.2)$ & & $20(12.0)$ & \\
\hline $\begin{array}{l}\text { Bachelor's degree } \\
\text { or higher }\end{array}$ & $6(2.8)$ & $1(16.7)$ & & $0(0.0)$ & & $0(0.0)$ & & $1(16.7)$ & & $1(16.7)$ & \\
\hline \multicolumn{12}{|l|}{ Occupation } \\
\hline Medical doctor & $7(3.3)$ & $2(28.5)$ & & $0(0.0)$ & & $0(0.0)$ & & $3(42.9)$ & & $3(42.9)$ & \\
\hline Nurse & $169(80.1)$ & $17(10.1)$ & 0.182 & $9(5.3)$ & 0.687 & $1(0.6)$ & 0.883 & $18(10.7)$ & $0.015^{*}$ & $23(13.6)$ & $0.029^{*}$ \\
\hline Others staff & 35 (16.6) & $2(5.7)$ & & $1(2.9)$ & & $0(0.0)$ & & $2(5.7)$ & & $2(5.7)$ & \\
\hline \multicolumn{12}{|l|}{ Department } \\
\hline Internal Medicine & $21(10.0)$ & $1(4.8)$ & 0.560 & $1(4.8)$ & 0.071 & $0(0.0)$ & 0.338 & $2(9.5)$ & 0.637 & $2(9.5)$ & 0.384 \\
\hline Surgical Department & $47(22.3)$ & $8(17.0)$ & & $1(2.1)$ & & $0(0.0)$ & & $8(17.0)$ & & $9(19.1)$ & \\
\hline Emergency Department & $16(7.6)$ & $1(6.3)$ & & $1(6.3)$ & & $0(0.0)$ & & $1(6.3)$ & & $1(6.3)$ & \\
\hline Paediatric & $19(9.0)$ & $2(10.5)$ & & $0(0.0)$ & & $1(5.3)$ & & $2(10.5)$ & & $4(21.1)$ & \\
\hline $\begin{array}{l}\text { Obstetrics and } \\
\text { Gynecology }\end{array}$ & $9(4.3)$ & $0(0.0)$ & & $0(0.0)$ & & $0(0.0)$ & & $0(0.0)$ & & $0(0.0)$ & \\
\hline Under Fives Clinic & $6(2.8)$ & $0(0.0)$ & & $1(16.7)$ & & $0(0.0)$ & & $0(0.0)$ & & $0(0.0)$ & \\
\hline Laboratory & $16(7.6)$ & $0(0.0)$ & & $0(0.0)$ & & $0(0.0)$ & & $0(0.0)$ & & $0(0.0)$ & \\
\hline OP Theatre & $22(10.4)$ & $2(9.0)$ & & $1(4.5)$ & & $0(0.0)$ & & $2(9.0)$ & & $2(9.0)$ & \\
\hline Pharmacy & $5(2.4)$ & $1(20.0)$ & & $2(40.0)$ & & $0(0.0)$ & & $1(20.0)$ & & $1(20.0)$ & \\
\hline Others & $50(23.7)$ & $6(12.0)$ & & $3(6.0)$ & & $0(0.0)$ & & $7(14.0)$ & & $9(18.0)$ & \\
\hline \multicolumn{12}{|l|}{ Hepatitis history } \\
\hline YES & $14(6.6)$ & $2(14.3)$ & 0.268 & $1(7.1)$ & 0.908 & $0(0.0)$ & 0.905 & $2(14.3)$ & 0.391 & $3(21.4)$ & 0.429 \\
\hline NO & $176(83.4)$ & $15(8.5)$ & & $8(4.5)$ & & $1(0.6)$ & & $17(9.7)$ & & $21(11.9)$ & \\
\hline Unknown & $21(10.0)$ & $4(19.0)$ & & $1(4.8)$ & & $0(0.0)$ & & $4(19.0)$ & & $4(19.0)$ & \\
\hline \multicolumn{12}{|l|}{ HBV vaccination } \\
\hline YES & 37 (17.5) & $1(2.7)$ & 0.135 & $6(16.2)$ & $0.002^{*}$ & $0(0.0)$ & 1.000 & $1(2.7)$ & 0.087 & $1(2.7)$ & $0.034^{*}$ \\
\hline NO & $174(82.5)$ & $20(11.5)$ & & $4(2.3)$ & & $1(0.6)$ & & $22(12.6)$ & & $27(15.5)$ & \\
\hline \multicolumn{12}{|l|}{ Sharps injury } \\
\hline Never & $110(52.1)$ & $11(10.0)$ & 0.955 & $5(4.5)$ & 0.739 & $1(1.0)$ & 0.630 & $14(12.7)$ & 0.586 & 15 (13.6) & 0.962 \\
\hline Once & $36(17.1)$ & $4(11.1)$ & & $1(2.8)$ & & $0(0.0)$ & & $4(11.1)$ & & $5(13.9)$ & \\
\hline More than once & $65(30.8)$ & $6(9.2)$ & & $4(6.2)$ & & $0(0.0)$ & & $5(7.7)$ & & $8(12.3)$ & \\
\hline Total & $211(100)$ & $21(10.0)$ & & $10(4.7)$ & & $1(0.5)$ & & $23(10.9)$ & & $28(13.3)$ & \\
\hline
\end{tabular}

"Statistically significant at $P<0.05$ 
carried out for seven possible risk factors, including age, gender, working experience years, education background, occupation, vaccination, and sharp injury history. Differences were considered statistically significant when the $p$ value was $<0.05$.

\section{Results}

\section{Demographic characteristics among HCWs}

The median age of the 211 HCWs who participated in the study was 39.0 years (range:18-59). Roughly half of participants were male (51.2\%), over the age of 39 (46.9\%), and had spent less than 9 years in their current job (46.4\%). The majority of HCWs had a middle education level (Diploma Certificate, 78.7\%) (Table 1).

\section{Prevalence of HBV}

Of the $211 \mathrm{HCWs}$, the positive detection rates of the five markers HBsAg, anti-HBs, HBeAg, anti-HBe and anti-HBc were 10.0, 4.7, 0.5, 10.9 and $13.3 \%$, respectively (Table 1). Twenty-one of the (10.0\%) HCWs tested positive for current $\mathrm{HBV}$ infections (HBsAg positive, anti-HBc positive) (Table 2), nine (4.3\%) were considered immune due to past infection (HBsAg negative and anti-HBc positive; anti-HBs positive), and nine (4.3\%) participants were immune due to hepatitis B vaccination (only anti-HBs positive). In total, 172 (81.5\%) participants were considered susceptible (all markers negative) (Table 2). Among 21 participants who were HBsAg positive, 13 (61.9\%) were HBV DNA positive. Twelve of them were determined very low-level HBV DNA $\left(<10^{\wedge} 3\right)$ and one had a DNA concentration of $5.6 \times 10^{\wedge} 3$ copies $/ \mathrm{mL}$.

Table 2 Summary of hepatitis B virus infection status among HCWs in the hospital, Sierra Leone

\begin{tabular}{ll}
\hline HBV infection classification & Number $(\%)(n=211)$ \\
\hline Susceptible & $172(81.5)$ \\
All markers negative & $172(81.5)$ \\
Acute or chronic infection & $21(10.0)$ \\
HBsAg (+), HBeAb (+), HBcAb (+) & $19(9.0)$ \\
HBsAg (+), HBeAb (+) & $1(0.5)$ \\
HBsAg (+), HBcAb (+) & $1(0.5)$ \\
Immune due to hepatitis B vaccination & $9(4.0)$ \\
Only HBsAb (+) & $9(4.3)$ \\
Immune due to natural infection & $9(4.0)$ \\
Only HBcAb (+) & $5(2.4)$ \\
HBeAb $(+), H B c A b(+)$ & $2(0.9)$ \\
HBsAb (+), HBeAb (+) & $1(0.5)$ \\
HBeAg $(+), H B c A b ~(+)$ & $1(0.5)$ \\
\hline
\end{tabular}

Anti-HBs positive rate of participants who reported receiving a $\mathrm{HB}$ vaccine significantly increased. This was in comparison to participants who had not received vaccination $(16.2 \%$ vs. $1.7 \%, p=0.001)$ (Table 2$)$. Of the 211 participants, only 14 (6.6\%) participants had clear HBV infection history, out of which 4 tested positive for serological markers (Table 2). The prevalence of the "current infection" group was significantly higher in HCWs $<39$ years old $(p=0.018)$ (Table 3). Multivariable factor analysis for risk for current HBV infection showed that there was a significantly lower risk for current HBV infection among those HCWs aged $>39$ years $(\mathrm{OR}=0.337 ; 95 \%$ CI:0.116-0.980; $p=0.046$ ) (Table 4).

\section{Knowledge of HBV infection and associated factors}

According to participants' responses, 77.3\% (163/211) of staff were not aware of clinical outcomes of HBV infection, while $63(29.9 \%)$ and $93(44.1 \%)$ had a poor knowledge on transmission routes and preventive measures of $\mathrm{HBV}$, respectively. The survey also revealed that working experience was associated with greater knowledge of preventive measures for $\operatorname{HBV}(p=0.017)$ and medical doctors were more knowledgeable about the consequences of HBV infection $(p=0.05)$ (Table 5).

\section{Discussion}

The global prevalence of hepatitis $\mathrm{B}$ is among the highest in parts of Africa, containing an estimated 50 million chronic carriers of HBV [13, 14]. Previous studies have shown HBsAg positive rates above $10 \%$ in African countries such as Burkina Faso, the Central African Republic, and Nigeria [15-17]. While in Sierra Leone reported HBsAg prevalence varied with different populations and times. Reports indicated a prevalence of $18 \%$ among children at a primary school in capital in 1998 [3], 6.2\% among pregnant women of middle and high socio-economic status in 2005 [4], and 13-15\% among blood donor candidates in Tonkolili District in 2017 [5]. In our present study, the sero-prevalence of HBsAg in HCWs was $10.0 \%$, which is similar to rates observed in Uganda [18], but higher than those in Nigeria (1.5\%) [19] and drastically higher than the developed European Region [20]. However, our study also found that the prevalence of anti-HBs was only $4.7 \%$ as compared to a South African report which detected a $19.9 \%$ anti-HBs positive rate in HCWs [21]. These results, especially the high prevalence of HBsAg and current HBV infection, suggest that hepatitis $\mathrm{B}$ is a very serious health concern in Sierra Leone. Our study also showed that the HBeAg positivity was low, but HBV DNA positivity was quite high (62\%). This may be indicative of the effects of $\mathrm{HBV}$ pre-C mutation in the study 
Table 3 Prevalence of four hepatitis B virus infection status by socio-demographic characteristics of the study participants

\begin{tabular}{|c|c|c|c|c|c|c|c|c|c|}
\hline Characteristic & $\begin{array}{l}\text { Tested } \\
\text { no. (\%) }\end{array}$ & $\begin{array}{l}\text { Susceptible } \\
\text { no. (\%) }\end{array}$ & $P$ value & $\begin{array}{l}\text { Current infection } \\
\text { no. (\%) }\end{array}$ & $P$ value & $\begin{array}{l}\text { Past infection } \\
\text { no. }(\%)\end{array}$ & $P$ value & $\begin{array}{l}\text { Immune due to } \\
\text { vaccination no. (\%) }\end{array}$ & $P$ value \\
\hline Age (y) & & & 0.337 & & $0.018^{*}$ & & 0.506 & & 0.506 \\
\hline$\geq 39 y$ & $112(53.1)$ & $94(83.9)$ & & $6(5.4)$ & & $6(5.4)$ & & $6(5.4)$ & \\
\hline$<39 y$ & $99(46.9)$ & 78 (78.8) & & $15(15.2)$ & & $3(3.0)$ & & $3(3.0)$ & \\
\hline Gender & & & 0.989 & & 0.730 & & 0.744 & & 0.499 \\
\hline Male & $108(51.2)$ & $88(81.5)$ & & $10(9.3)$ & & $4(3.7)$ & & $6(5.6)$ & \\
\hline Female & $103(48.8)$ & $84(81.6)$ & & $11(10.7)$ & & $5(4.9)$ & & $3(2.9)$ & \\
\hline Working experience & & & 0.452 & & 0.419 & & 0.509 & & 0.736 \\
\hline$\geq 9 y$ & $113(53.6)$ & 90 (79.6) & & $13(11.5)$ & & $6(5.3)$ & & $4(3.5)$ & \\
\hline$<9 y$ & $98(46.4)$ & $82(83.7)$ & & $8(8.2)$ & & $3(3.1)$ & & $5(5.1)$ & \\
\hline Education level & & & 0.933 & & 0.365 & & 0.716 & & 0.716 \\
\hline High school & 39 (18.5) & $31(79.5)$ & & $6(15.4)$ & & $1(2.6)$ & & $1(2.6)$ & \\
\hline Diploma certificate & $166(78.7)$ & $136(81.9)$ & & $14(8.4)$ & & $8(4.8)$ & & $8(4.8)$ & \\
\hline $\begin{array}{l}\text { Bachelor's degree } \\
\text { or higher }\end{array}$ & $6(2.8)$ & $5(83.3)$ & & $1(16.7)$ & & $0(0.0)$ & & $0(0.0)$ & \\
\hline Occupation & & & 0.736 & & 0.894 & & 0.311 & & 0.780 \\
\hline Medical doctor & $7(3.3)$ & $6(85.7)$ & & $1(14.3)$ & & $0(0.0)$ & & $0(0.0)$ & \\
\hline Nurse & $169(80.1)$ & $136(80.5)$ & & $17(10.1)$ & & $9(5.3)$ & & $7(4.1)$ & \\
\hline Other staff & 35 (16.6) & $30(85.7)$ & & $3(8.6)$ & & $0(0.0)$ & & $2(5.7)$ & \\
\hline Department & & & $0.000^{*}$ & & 0.560 & & 0.738 & & $0.007^{*}$ \\
\hline Internal medicine & $21(10.0)$ & $18(85.7)$ & & $1(4.8)$ & & $1(4.8)$ & & $1(4.8)$ & \\
\hline Surgical Department & $47(22.3)$ & $36(76.6)$ & & $8(17.0)$ & & $3(6.4)$ & & $0(0.0)$ & \\
\hline Emergency Department & $16(7.6)$ & $14(87.5)$ & & $1(6.3)$ & & $0(0.0)$ & & $1(6.3)$ & \\
\hline Pediatric & $19(9.0)$ & $15(78.9)$ & & $2(10.5)$ & & $2(10.5)$ & & $0(0.0)$ & \\
\hline $\begin{array}{l}\text { Obstetrics and } \\
\text { Gynecology }\end{array}$ & $9(4.3)$ & $0(0.0)$ & & $0(0.0)$ & & $0(0.0)$ & & $0(0.0)$ & \\
\hline Under Fives Clinic & $6(2.8)$ & $5(83.3)$ & & $0(0.0)$ & & $0(0.0)$ & & $1(16.7)$ & \\
\hline Laboratory & $16(7.6)$ & $16(100.0)$ & & $0(0.0)$ & & $0(0.0)$ & & $0(0.0)$ & \\
\hline OP Theatre & $22(10.4)$ & $19(40.9)$ & & $2(9.0)$ & & $0(0.0)$ & & $1(4.5)$ & \\
\hline Pharmacy & $5(2.4)$ & $2(40.0)$ & & $1(20.0)$ & & $0(0.0)$ & & $2(40.0)$ & \\
\hline Others & $50(23.7)$ & $38(76.0)$ & & $6(12.0)$ & & $3(6.0)$ & & $3(6.0)$ & \\
\hline Hepatitis history & & & 0.355 & & 0.854 & & 0.110 & & 0.393 \\
\hline YES & $14(6.6)$ & $10(71.4)$ & & $2(14.3)$ & & $2(14.3)$ & & $0(0.0)$ & \\
\hline NO & $176(83.4)$ & 143 (81.3) & & $17(9.7)$ & & $7(4.0)$ & & $9(5.1)$ & \\
\hline Unknown & $21(10.0)$ & 19 (90.5) & & $2(9.5)$ & & $0(0.0)$ & & $0(0.0)$ & \\
\hline HBV vaccination & & & 0.588 & & 0.135 & & 1.000 & & $0.001^{*}$ \\
\hline YES & $37(17.5)$ & 29 (78.4) & & $1(2.7)$ & & $1(2.7)$ & & $6(16.2)$ & \\
\hline NO & $174(82.5)$ & $143(82.2)$ & & $20(11.5)$ & & $8(4.6)$ & & $3(1.7)$ & \\
\hline Sharps injury & & & $0.025^{*}$ & & 0.351 & & 0.670 & & 0.063 \\
\hline Never & $110(52.1)$ & $82(74.5)$ & & $14(12.7)$ & & $6(5.5)$ & & $8(7.3)$ & \\
\hline Once & $36(17.1)$ & 32 (88.9) & & $2(5.6)$ & & $1(2.8)$ & & $1(2.8)$ & \\
\hline More than once & 65 (30.8) & $58(89.2)$ & & $5(7.7)$ & & $2(3.1)$ & & $0(0.0)$ & \\
\hline Total & $211(100)$ & $172(81.5)$ & & $21(10.0)$ & & $9(4.3)$ & & $9(4.3)$ & \\
\hline
\end{tabular}

"Statistically significant at $P<0.05$ 
Table 4 Multivariable analysis of possible risk factors for current HBV infection

\begin{tabular}{|c|c|c|c|c|c|}
\hline Variables & Category & Frequency & OR & $95 \% \mathrm{Cl}$ for OR & $P$ Value \\
\hline \multirow[t]{2}{*}{ Age } & $\geq 39 y$ & $6 / 112$ & \multirow[t]{2}{*}{0.337} & \multirow[t]{2}{*}{$0.116-0.980$} & \multirow[t]{2}{*}{$0.046^{*}$} \\
\hline & $<39 y$ & $15 / 99$ & & & \\
\hline \multirow[t]{2}{*}{ Gender } & Male & 10/108 & \multirow[t]{2}{*}{1.304} & \multirow[t]{2}{*}{$0.471-3.609$} & \multirow[t]{2}{*}{0.609} \\
\hline & Female & $11 / 103$ & & & \\
\hline \multirow{2}{*}{$\begin{array}{l}\text { Working } \\
\text { experience }\end{array}$} & $\geq 9 y$ & $13 / 113$ & \multirow[t]{2}{*}{1.334} & \multirow[t]{2}{*}{$0.502-3.547$} & \multirow[t]{2}{*}{0.563} \\
\hline & $<9 y$ & $8 / 98$ & & & \\
\hline \multirow{3}{*}{$\begin{array}{l}\text { Education } \\
\text { background }\end{array}$} & Low & $6 / 39$ & \multirow[t]{3}{*}{0.401} & \multirow[t]{3}{*}{$0.104-1.547$} & \multirow[t]{3}{*}{0.185} \\
\hline & Mid & $14 / 166$ & & & \\
\hline & High & $1 / 6$ & & & \\
\hline \multirow[t]{3}{*}{ Occupation } & Doctors & $1 / 7$ & \multirow[t]{3}{*}{2.114} & \multirow[t]{3}{*}{$0.496-9.018$} & \multirow[t]{3}{*}{0.312} \\
\hline & Nurses & $17 / 169$ & & & \\
\hline & Others & $3 / 35$ & & & \\
\hline \multirow[t]{2}{*}{ Vaccination } & Yes & $1 / 37$ & \multirow[t]{2}{*}{0.291} & \multirow[t]{2}{*}{$0.036-2.383$} & \multirow[t]{2}{*}{0.250} \\
\hline & No & $20 / 174$ & & & \\
\hline \multirow[t]{3}{*}{ Sharps injury } & Never & $14 / 110$ & \multirow[t]{3}{*}{0.692} & \multirow[t]{3}{*}{$0.391-1.225$} & \multirow[t]{3}{*}{0.207} \\
\hline & Once & $2 / 36$ & & & \\
\hline & $\begin{array}{l}\text { More } \\
\text { than once }\end{array}$ & $5 / 65$ & & & \\
\hline
\end{tabular}

"Statistically significant at $P<0.05$ population, another issues which requires further investigation.

A significant difference in current infection rate between participants younger than 39 years old $(p=0.018)$ was observed in this study. Additionally, multivariable analysis of possible risk factors suggests a lower risk for current HBV infection among those $\mathrm{HCWs}$ aged $>39$ years $(\mathrm{OR}=0.337$; 95\% CI:0.116-0.980; $p=0.046)$. This finding may be due to differences in lifestyle or behavior between the two groups, however a larger sample size is needed to adequately study this risk factor. As expected, we also found that HBV vaccination was a protective factor for anti-HBs positive (immune status due to vaccination), as demonstrated elsewhere [22]. Before the vaccination program was launched in 1995, HBsAg carriage in the African population was very high [23]. In Sierra Leone, the hepatitis $B$ vaccine is not available for the entire population because of limited resources. Our study found that only 17.5\% HCWs reported previous HBV vaccination history, which is higher than those in the Democratic Republic of Congo (3.6\%) [10] and Ethiopia (5.4\%) [24]. However, only $16.2 \%$ of those vaccinated produced protective antibodies against HBV. This may have resulted from receiving a vaccination many years ago thereby resulting in waning immunity. In addition, there were three individuals who did not report previous HBV vaccination, but had similar

Table 5 Responses of the study participants to basic hepatitis B knowledge

\begin{tabular}{|c|c|c|c|c|c|c|c|c|c|c|c|c|}
\hline \multirow[t]{3}{*}{ Characteristic } & \multicolumn{4}{|c|}{ The consequences of infection } & \multicolumn{4}{|c|}{ Route of transmission } & \multicolumn{4}{|c|}{ Preventive measures } \\
\hline & \multicolumn{3}{|l|}{ no. (\%) } & \multirow{2}{*}{$\begin{array}{l}P \\
\text { value }\end{array}$} & \multicolumn{3}{|c|}{ no. (\%) } & \multirow{2}{*}{$\begin{array}{l}P \\
\text { value }\end{array}$} & \multicolumn{3}{|l|}{ no. (\%) } & \multirow{2}{*}{$\begin{array}{l}P \\
\text { value }\end{array}$} \\
\hline & Poor & Intermed & Good & & Poor & Intermed & Good & & Poor & Intermed & Good & \\
\hline \multicolumn{13}{|l|}{ Age (y) } \\
\hline$<39 y$ & $77(77.8)$ & $15(15.2)$ & $7(7.1)$ & \multirow[t]{2}{*}{0.920} & $31(31.3)$ & $35(35.4)$ & $33(33.3)$ & \multirow[t]{2}{*}{0.896} & $41(41.4)$ & $17(17.2)$ & $41(41.4)$ & \multirow[t]{2}{*}{0.736} \\
\hline$\geq 39 y$ & $86(76.8)$ & 19 (16.9) & $7(6.3)$ & & $32(28.6)$ & $40(35.7)$ & $40(35.7)$ & & $52(46.4)$ & 19 (16.9) & $41(36.7)$ & \\
\hline \multicolumn{13}{|l|}{ Gender } \\
\hline Female & $75(72.8)$ & $20(19.4)$ & $8(7.8)$ & \multirow[t]{2}{*}{0.322} & $26(25.4)$ & $42(40.8)$ & $35(33.9)$ & \multirow[t]{2}{*}{0.222} & $42(41.7)$ & $19(18.5)$ & $42(40.8)$ & \multirow[t]{2}{*}{0.634} \\
\hline Male & $88(81.5)$ & $14(12.9)$ & $6(5.6)$ & & $37(34.3)$ & $33(30.6)$ & $38(35.2)$ & & $51(47.2)$ & $17(15.7)$ & $40(37.0)$ & \\
\hline \multicolumn{13}{|l|}{ Working experience } \\
\hline$<9 y$ & 74 (75.5) & $19(19.4)$ & $5(5.1)$ & \multirow[t]{2}{*}{0.380} & $27(27.6)$ & $35(35.7)$ & $36(36.7)$ & \multirow[t]{2}{*}{0.752} & $33(33.7)$ & $19(19.4)$ & $46(46.9)$ & \multirow[t]{2}{*}{$0.017^{*}$} \\
\hline$\geq 9 y$ & 89 (78.8) & $15(13.3)$ & $9(7.9)$ & & 36 (31.9) & $40(35.4)$ & $37(32.7)$ & & $60(53.1)$ & $17(15.0)$ & 36 (31.9) & \\
\hline \multicolumn{13}{|l|}{ Education level } \\
\hline High school & $25(65.8)$ & $9(23.7)$ & $4(10.5)$ & \multirow[t]{3}{*}{0.324} & $9(20.5)$ & $16(41.0)$ & $13(33.3)$ & \multirow[t]{3}{*}{0.378} & $15(38.5)$ & $9(23.1)$ & $14(35.9)$ & \multirow[t]{3}{*}{0.324} \\
\hline Diploma certificate & $133(79.6)$ & $24(14.4)$ & $10(5.9)$ & & $51(31.7)$ & $58(35.4)$ & $58(35.4)$ & & $75(45.7)$ & $26(15.9)$ & $66(40.2)$ & \\
\hline $\begin{array}{l}\text { Bachelor's degree } \\
\text { or higher }\end{array}$ & $5(83.3)$ & $1(16.7)$ & $0(0.0)$ & & $3(37.5)$ & $1(12.5)$ & $2(25.0)$ & & $3(50.0)$ & $1(16.7)$ & $2(33.3)$ & \\
\hline \multicolumn{13}{|l|}{ Occupation } \\
\hline Medical doctor & $4(57.1)$ & $2(28.6)$ & $1(14.3)$ & \multirow[t]{4}{*}{$0.050^{*}$} & $0(0.0)$ & $3(42.9)$ & $4(57.1)$ & \multirow[t]{4}{*}{0.196} & $2(28.6)$ & $1(14.3)$ & $4(57.1)$ & \multirow[t]{4}{*}{0.221} \\
\hline Nurse & $137(81.1)$ & $21(12.4)$ & $11(6.5)$ & & $56(33.1)$ & 59 (34.9) & $54(31.9)$ & & $80(47.3)$ & $30(17.8)$ & $59(34.9)$ & \\
\hline Other staff & $22(62.9)$ & $11(31.4)$ & $2(5.7)$ & & $7(20.0)$ & $13(37.1)$ & $15(42.9)$ & & $11(31.4)$ & $5(14.3)$ & $19(54.3)$ & \\
\hline Total & $163(77.3)$ & $34(16.1)$ & $14(6.6)$ & & $63(29.9)$ & 75 (35.5) & 73 (34.6) & & $93(44.1)$ & $36(17.1)$ & $82(38.9)$ & \\
\hline
\end{tabular}

"Statistically significant at $P<0.05$ 
immunological results to those who had been vaccinated, which was likely a result of recall bias. Vaccination of HCWs for HBV has been recommended by the WHO. However, even in South Africa, where there is a stronger healthcare system, only $30.6-52.4 \%$ of HCWs had protective levels of anti-HBs [7]. In addition, $81.5 \%$ HCWs in Sierra Leone tested negative for all markers, indicating susceptibility to HBV infection. Thus, there is an urgent need to expand vaccination coverage rates among HCWs in Sierra Leone.

The present study also found that there was poor knowledge of $\mathrm{HBV}$, including the clinical outcome of infection, route of transmission and preventive measures of HBV, among HCWs. Therefore, HCWs in Sierra Leone will continue to be at risk of HBV infections until training and vaccination programs are strengthened. Our study also demonstrates that HCWs with longer working experience had more knowledge about preventive measures as compared to those with less work experience $(p=0.007)$. Furthermore, medical doctors had more knowledge of the consequences of HBV infection $(p=0.05)$, as expected compared to other occupations. It is generally assumed that education level and departments correlate with overall knowledge levels of the infection. However, this was not the case as these factors were not significant across all categories of knowledge, which is likely due to the small sample size of specific departments and education levels.

\section{Limitations}

The data presented in this study comes from a single hospital, which may not be representative of other healthcare facilities in Sierra Leone. It would be premature to draw broader conclusions regarding the prevalence and knowledge of hepatitis B across all HCWs in Sierra Leone.

\section{Conclusions}

HCWs in Sierra Leone lacked adequate knowledge of the hepatitis B virus.. Additionally, there were low coverage rates of hepatitis $B$ vaccination that does not seem to be able to meet the WHO recommendations, leaving many HCWs susceptible to hepatitis B infection in the sampled population. Local health authorities need to make a coordinated effort to increase vaccination uptake considering the cost-effectiveness of broad immunization against hepatitis $\mathrm{B}$ and incorporate more intensive training against blood-borne pathogens for HCWs in Sierra Leone.

\section{Additional file}

Additional file 1: The original data of HBV surveillance for HCWs from Sierra Leone. (XLSX $25 \mathrm{~kb}$ )

\section{Abbreviations}

DNA: Deoxyribonucleic acid; HBV: Hepatitis B virus; HCWs: Healthcare workers; WHO: World Health Organization

\section{Acknowledgements}

We are thankful to all healthcare workers who volunteered their valuable information and to the Public Health Department for their great assistance in organization this investigation and collecting the data. The authors also express gratitude to Dr. Michael E. von Fricken from George Mason University for editing the manuscript. Chinese Military Medical Experts Group in Sierra Leone: Yu-Ling Qin, Bo Li, Yue Yuan, Yi Sun, Jing Li, Zhong-Peng Zhao, Jun Jiao, Ya-Jun Song, Tian-Jun Jiang, Jia-Fu Jiang.

\section{Funding}

This project was supported by a training grant by Beijing 302 Hospital, of which YLQ and JFJ were coprincipal investigators.

\section{Availability of data and materials}

All data generated or analysed during this study are included in this published article and the Additional file 1.

\section{Authors' contributions}

YLQ, BL XZ, and TJJ conceived the survey. YLQ, BL, YY, YSZ, SB, LL, PL and TJJ designed and created the questionnaire. BL, YJS, YY, JL, ZPZ, JJ, SS, YS, JEK and TJJ conducted the survey. JFJ, BL, YLQ and YY executed data collection and managing and statistical analysis. BL and JJF drafted the manuscript. FS supervised this work. All authors critically revised the article and approved the final version of the manuscript.

\section{Ethics approval and consent to participate}

This work was conducted as part of the cooperation in military medicine by Chinese Military Medical Experts Group in Sierra Leone, with all activities coordinated by the Joint Medical Unit of the Republic of Sierra Leone Armed Forces. The ethics committees of the No. 302 Hospital, Beijing, China, approved this study. Written informed consent was obtained from participants who tested for the HBV and respond to the survey questionnaire. The survey was anonymous and did not collect any personal data that could lead to identification of survey participants.

Consent for publication

Not applicable.

\section{Competing interests}

The authors declare that they have no competing interests.

\section{Publisher's Note}

Springer Nature remains neutral with regard to jurisdictional claims in published maps and institutional affiliations.

\section{Author details}

${ }^{1}$ Beijing 302 Hospital, Beijing, China. ${ }^{2}$ Beijing Institute of Microbiology and Epidemiology, Beijing, China. ${ }^{3}$ No. 34 Military Hospital, Wilberforce, Freetown, Sierra Leone. ${ }^{4}$ College of Medicine and Allied Health Sciences, Freetown, Sierra Leone.

Received: 16 January 2018 Accepted: 3 July 2018

Published online: 09 July 2018

References

1. World Health Organization. Global Hepatitis Report. 2017. Available at: http:// apps.who.int/iris/bitstream/10665/255016/1/9789241565455-eng.pdf?ua=1 .

2. Turati F, Edefonti V, Talamini R, Ferraroni M, Malvezzi M, Bravi F, et al. Family history of liver cancer and hepatocellular carcinoma. Hepatology. 2012;55(5):1416-25.

3. Hodges M, Sanders E, Aitken C. Seroprevalence of hepatitis markers; HAV, HBV, HCV and HEV amongst primary school children in Freetown, Sierra Leone. West Afr J Med. 1998;17:36-7.

4. Wurie IM, Wurie AT, Gevao SM. Sero-prevalence of hepatitis B virus among middle to high socio-economic antenatal population in Sierra Leone. West Afr J Med. 2005;24(1):18-20. 
5. Garcia-Tardon N, Gresnigt TM, Fofanah AB, Grobusch MP. Hepatitis B and C in Tonkolili Province, Sierra Leone. Lancet. 2017;390(10101):1485.

6. World Health Organization. Preventing perinatal hepatitis B virus transmission: a guide for introducing and strengthening hepatitis B birth dose vaccination. Geneva: World Health Organization; 2015.

7. Sondlane TH, Mawela L, Razwiedani LL, Selabe SG, Lebelo RL, Nare Rakgole J, et al. High prevalence of active and occult hepatitis B virus infections in healthcare workers from two provinces of South Africa. Vaccine. 2016:34(33):3835-9.

8. Alese $\mathrm{OO}$, Alese MO, Ohunakin A, Oluyide PO. Seroprevalence of hepatitis B surface antigen and occupational risk factors among health care workers in Ekiti state. Nigeria J Clin Diagn Res. 2016;10(2):LC16-8.

9. Coppola N, De Pascalis S, Onorato L, Calò F, Sagnelli C, Sagnelli E. Hepatitis $B$ virus and hepatitis $C$ virus infection in healthcare workers. World J Hepatol. 2016:8(5):273-81.

10. Shindano TA, Bahizire E, Fiasse R, Horsmans Y. Knowledge, attitudes, and practices of health-care workers about viral hepatitis $B$ and $C$ in south Kivu. Am J Trop Med Hyg. 2017;96(2):400-4

11. Topuridze M, Butsashvili M, Kamkamidze G, Kajaia M, Morse D, McNutt LA. Barriers to hepatitis B vaccine coverage among healthcare workers in the republic of Georgia: an international perspective. Infect Control Hosp Epidemiol. 2010;31(2):158-64

12. Winchester SA, Tomkins S, Cliffe S, Batty L, Ncube F, Zuckerman M. Healthcare workers' perceptions of occupational exposure to blood-borne viruses and reporting barriers: a questionnaire-based study. J Hosp Infect. 2012;82(1):36-9.

13. Ott JJ, Stevens GA, Groeger J, Wiersma ST. Global epidemiology of hepatitis $B$ virus infection: new estimates of age-specific HBsAg seroprevalence and endemicity. Vaccine. 2012;30:2212-9.

14. Aba HO, Aminu M. Seroprevalence of hepatitis B virus serological markers among pregnant Nigerian women. Ann Afr Med. 2016;15(1):20-7.

15. Komas NP, Vickos U, Hubschen JM, Bere A, Manirakiza A, Muller CP, et al. Cross-sectional study of hepatitis B virus infection in rural communities, Central African Republic. BMC Infect Dis. 2013;13:286.

16. Tao I, Compaore TR, Diarra B, Djigma F, Zohoncon TM, Assih M, et al. Seroepidemiology of hepatitis $B$ and $C$ viruses in the general population of Burkina Faso. Hepat Res Treat. 2014:781843.

17. Musa BM, Bussell S, Borodo MM, Samaila AA, Femi OL. Prevalence of hepatitis B virus infection in Nigeria, 2000-2013: a systematic review and meta-analysis. Niger J Clin Pract. 2015;18:163-72.

18. Ziraba AK, Bwogi J, Namale A, Wainaina CW, Mayanja-Kizza H. Seroprevalence and risk factors for hepatitis B virus infection among health care workers in a tertiary hospital in Uganda. BMC Infect Dis. 2010;29(10):191.

19. Abiola AH, Agunbiade AB, Badmos KB, Lesi AO, Lawal AO, Alli QO Prevalence of $\mathrm{HBsAg}$, knowledge, and vaccination practice against viral hepatitis B infection among doctors and nurses in a secondary health care facility in Lagos state, South-Western Nigeria. Pan Afri Med J. 2016;23:160.

20. Hope VD, Eramova I, Capurro D, Donoghoe MC. Prevalence and estimation of hepatitis $B$ and $C$ infections in the WHO European region: a review of data focusing on the countries outside the European Union and the European free trade association. Epidemiol Infect. 2014;142:270-86.

21. Burnett RJ, François G, Mphahlele MJG, Africa PN, Satekge MM, et al. Hepatitis B vaccination coverage in healthcare workers in Gauteng Province, South Africa. Vaccine. 2011;29(25):4293-7.

22. Ghadir MR, Belbasi M, Heidari A, et al. Distribution and risk factors of hepatitis B virus infection in the general population of Central Iran. Hepat Mon. 2012;12:112-7.

23. Burnett RJ, François G, Kew MC, Leroux-Roels G, Meheus A, Hoosen AA, et al. Hepatitis $B$ virus and human immunodeficiency virus co-infection in subSaharan Africa: a call for further investigation. Liver Int. 2005;25(2):201-13.

24. Abeje G, Azage M. Hepatitis B vaccine knowledge and vaccination status among health care workers of Bahir Dar City Administration, Northwest Ethiopia: a cross sectional study. BMC Infect Dis. 2015;15:30,

\section{Ready to submit your research? Choose BMC and benefit from:}

- fast, convenient online submission

- thorough peer review by experienced researchers in your field

- rapid publication on acceptance

- support for research data, including large and complex data types

- gold Open Access which fosters wider collaboration and increased citations

- maximum visibility for your research: over $100 \mathrm{M}$ website views per year

At BMC, research is always in progress.

Learn more biomedcentral.com/submissions 